\title{
GENERALIZATION OF SCHWARZ-PICK LEMMA TO INVARIANT VOLUME IN A KÄHLER MANIFOLD
}

BY KYONG T. HAHN AND JOSEPHINE MITCHELL

Communicated by Paul T. Bateman, March 27, 1967

Let $D$ be the class of bounded homogeneous domains $D$ in the space $C^{n}$ of $n$ complex variables $z=\left(z^{1}, \cdots, z^{n}\right)$. A domain $D$ is homogeneous if any point of $D$ can be mapped into any other by a holomorphic automorphism. A bounded domain $D$ possesses the Bergman metric, which is invariant under biholomorphic mappings of $D$, given by

$$
d s_{D}^{2}=T_{\alpha \bar{\beta}} d z^{\alpha} d \bar{z}^{\beta}
$$

(the summation convention is used), where

$$
\begin{aligned}
T_{\alpha \bar{\beta}} & =T_{\alpha \bar{\beta}}(z, \bar{z})=\left(\partial^{2} \log K_{D}\right) /\left(\partial z^{\alpha} \partial \bar{z}^{\beta}\right), \\
T_{D} & =T_{D}(z, \bar{z})=\operatorname{det}\left(T_{\alpha \bar{\beta}}\right),
\end{aligned}
$$

and $K_{D}=K_{D}(z, \bar{z})$ is the Bergman kernel function of $D$ [2]. The functions $K_{D}(z, \bar{z})$ and $T_{D}(z, \bar{z})$ are relative invariants of $D$ under biholomorphic mappings and consequently the function

$$
I_{D}(z, \bar{z})=K_{D}(z, \bar{z}) / T_{D}(z, \bar{z})
$$

is an invariant of $D$. The kernel function $K_{D}(z, \bar{z})$ becomes infinite on the boundary of $D$.

Let $\mathfrak{K}$ be the class of Kähler manifolds $\Delta$ with metric given by

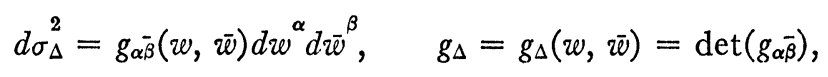

where $w$ is a local coordinate of a point on $\Delta$. We also assume

$$
\begin{gathered}
-r_{\alpha \bar{\beta}} u^{\alpha} \bar{u}^{\beta} \geqq 0 \quad \text { for any vector } u=\left(u^{\alpha}\right), \\
\operatorname{det}\left(-r_{\alpha \bar{\beta}}\right) \geqq g_{\Delta},
\end{gathered}
$$

where $r_{\alpha \bar{\beta}}=-\left(\partial^{2} \log g_{\Delta}\right) /\left(\partial w^{\alpha} \partial \bar{w}^{\beta}\right)$ are the components of the Ricci curvature tensor of the metric (4).

A domain $D$ is star-like with respect to a point $z_{0} \in D$ if $z \in D$ implies $r\left(z-z_{0}\right) \in D$ for $0<r \leqq 1$. If $D$ is star-like, then the image domains $D_{r}$ of $D$ under the similarity map

$$
w=r\left(z-z_{0}\right), \quad 0<r \leqq 1
$$


are such that $D_{r_{1}} \subset D_{r_{2}}$ if $r_{1} \leqq r_{2}$, and $D=\bigcup_{j=1}^{\infty} D_{r_{j}}$, where $r_{j}, 0<r_{j}<1$, is an increasing sequence with limit 1.

THEOREM 1. If DED is star-like and can be mapped biholomorphically by $w=w(z)$ into a Kähler manifold $\Delta \in \mathcal{K}$, then

$$
g_{\Delta}(w, \bar{w})\left|J_{w}(z)\right|^{2} \leqq T_{D}(z, \bar{z})
$$

on $D$, where $J_{w}(z)$ is the Jacobian of $w=w(z)$.

Proof (Sketch). For any $z \in D$, there exists an $r$ such that $z \in D_{r}$. Let

$$
G_{\alpha \bar{\beta}} d z^{\alpha} d \bar{z}^{\beta}
$$

be the hermitian form on $D$ corresponding to the metric (4) on $\Delta \in \Re$ under the inverse mapping $z=z(w)$ of $w: D \rightarrow \Delta$. Then

$$
G_{D}(z, \bar{z})=\operatorname{det}\left(G_{\alpha \bar{\beta}}\right)=g_{\Delta}(w, \bar{w})\left|J_{w}(z)\right|^{2}>0
$$

and

$$
\begin{aligned}
\operatorname{det}\left(-R_{\alpha \bar{\beta}}\right) & \geqq g_{\Delta}(w, \tau \bar{w})\left|J_{w}(z)\right|^{2}=G_{D}(z, \bar{z}), \\
R_{\alpha \bar{\beta}} & =R_{\alpha \bar{\beta}}(z, \bar{z})=-\left(\partial^{2} \log G_{D}\right) /\left(\partial z^{\alpha} \partial \bar{z}^{\beta}\right) .
\end{aligned}
$$

Let $U=\log \left(G_{D}(z, \bar{z}) / T_{D}(0,0)\right), V=\log \left(K_{D_{r}}(z, \bar{z}) / K_{D}(0,0)\right)$. By a similar argument to that of Dinghas and Ahlfors [1], [3] we show that $U \leqq V$ on $D_{r}$, from which Theorem 1 follows.

Let $S$ be a homogeneous Siegel domain of second kind. There exists an increasing sequence $\left\{S_{\nu}\right\}$ of homogeneous subdomains with limit $S$ and such that $S_{\nu} \cup \partial S_{\nu} \subset S_{\nu+1}$, where $\partial S_{\nu}$ is the boundary of $S_{\nu}$ lying in (finite) $C^{n}$ space. Let $K^{\prime}$ be that subclass of $\Re$ for which the metric can be chosen so that

$$
\lim _{\zeta \rightarrow \zeta_{\infty}} G_{S}(\zeta, \bar{\zeta}) / T_{S \nu}(\zeta, \bar{\zeta}) \leqq 1, \quad \zeta \in S_{\nu},
$$

for all $\nu$ sufficiently large where $\zeta_{\infty}$ is a boundary point of $S_{\nu}$ which is "a point at infinity." The class $\mathcal{K}^{\prime}$ is nonempty. An extension of Theorem 1 to domains $S$ is given in

Theorem 2. Let $S$ be a homogeneous Siegel domain of second kind. If $w=w(\zeta)$ maps $S$ biholomorphically into a Kähler manifold $\Delta \in K^{\prime}$, then $g_{\Delta}(w, \bar{w})\left|J_{w}(\zeta)\right|^{2} \leqq T_{S}(\zeta, \bar{\zeta})$ on $S$.

Then by a well-known result of Vinberg, Gindikin and Pjatecki1Sapiro [7] that every bounded homogeneous domain $D$ can be mapped biholomorphically onto an affinely homogeneous Siegel domain of second kind we have 
TheOREM 3. If $D \in D$ can be mapped into a Kähler manifold $\Delta$ in $\mathfrak{K}^{\prime}$ by a biholomorphic mapping $w=w(z)$, then for $z \in D$,

$$
g_{D}(w, \bar{w})\left|J_{w}(z)\right|^{2} \leqq T_{D}(z, \bar{z}) .
$$

Proof of Theorem 2 (Sketch). By the result of Pjateckiř-Sapiro [6] that the Siegel domain of second kind is biholomorphically equivalent to a bounded domain $D$ we get an increasing sequence of homogeneous bounded subdomains $D_{\nu}$, with union $D$, corresponding to the sequence $\left\{S_{\nu}\right\}$ for $S$. Then an analogous argument to that in Theorem 1 is applicable.

REMARK. Full details will appear in [4] and [5].

\section{REFERENCES}

1. L. V. Ahlfors, An extension of Schwarz's lemma, Trans. Amer. Math. Soc. 43 (1938), 359-364.

2. S. Bergman, Sur les fonctions orthogonales de plusiers variables complexes avec les applications à la theorie des fonctions analytiques, Mem. Sci. Math. 106 (1947), Paris.

3. A. Dinghas, "Ein N-dimensionalen Analogen des Schwarz-Pickschen Flächensatzes für holomorphe Abbildungen der komplexen Einheitskugel in eine Kähler Mannigfaltigkeit" in Festschrift zur Gedachtnisfeier für Karl Weierstrass 1815-1965, Wiss. Abh. Arbeitsgemeinschaft Forsch. des Landes Nordr.-Westfalen, Bd. 33, Westdeutscher Verlag, Cologne, 1966.

4. K. T. Hahn and Josephine Mitchell, Generalization of Schrwarz-Pick lemma to invariant volume in Kähler manifold, Trans Amer. Math. Soc. 127 (1967).

5. - Generalization of Schwarz-Pick lemma to invariant volume in a Kähler manifold. II, (to appear).

6. I. I. Pjateckil-Sapiro, Geometry of classical domains and theory of automorphic functions, Fizmatgiz, Moscow, 1961.

7. E. B. Vinberg, S. G. Gindikin and I. I. Pjateckilı-Sapiro, Classification and canonical realization of complex homogeneous bounded domains, Trudy Moscow, Mat. Obsc. 12 (1963), 359-388, Trans. Moscow Math. Soc. 12 (1963), 404-437.

The Pennsylvania State University 